\title{
Fractional equations of Curie-von Schweidler and Gauss laws
}

\author{
Vasily E Tarasov \\ Skobeltsyn Institute of Nuclear Physics, Moscow State University, Moscow 119991, Russia \\ E-mail: tarasov@ theory.sinp.msu.ru
}

Received 10 October 2007, in final form 31 January 2008

Published 19 March 2008

Online at stacks.iop.org/JPhysCM/20/145212

\begin{abstract}
The dielectric susceptibility of most materials follows a fractional power-law frequency dependence that is called the 'universal' response. We prove that in the time domain this dependence gives differential equations with derivatives and integrals of noninteger order. We obtain equations that describe 'universal' Curie-von Schweidler and Gauss laws for such dielectric materials. These laws are presented by fractional differential equations such that the electromagnetic fields in the materials demonstrate 'universal' fractional damping. The suggested fractional equations are common (universal) to a wide class of materials, regardless of the type of physical structure, chemical composition, or of the nature of the polarization.
\end{abstract}

\section{Introduction}

A growing number of dielectric relaxation data show that the classical Debye behavior [1-3] is hardly ever observed experimentally [4-7]. Instead it has been derived [4-7] that power laws are a common feature of the dielectric response of most materials for wide frequency ranges. The fact that different dielectric spectra are described by the power laws is confirmed in many measurements [4-6] for a wide class of various substances. The dielectric susceptibility of most materials follows, over extended frequency ranges, a fractional power-law frequency dependence, which is called the law of 'universal' response [4-6]. This law is found both in dipolar materials beyond their loss-peak frequency, and in materials where the polarization arises from movements of either ionic or electronic hopping charge carriers. These power-law responses are most easily displayed in terms of the dielectric susceptibility $\tilde{\chi}(\omega)=\chi^{\prime}(\omega)-\mathrm{i} \chi^{\prime \prime}(\omega)$ as a function of frequency $\omega$. It has been found $[8,9]$ that the frequency dependence of the dielectric susceptibility follows a common universal pattern for virtually all kinds of materials. The behavior

$$
\begin{gathered}
\chi^{\prime}(\omega) \sim \omega^{n-1}, \quad \chi^{\prime \prime}(\omega) \sim \omega^{n-1}, \\
\left(0<n<1, \omega \gg \omega_{\mathrm{p}}\right),
\end{gathered}
$$

and

$$
\begin{gathered}
\chi^{\prime}(0)-\chi^{\prime}(\omega) \sim \omega^{m}, \quad \chi^{\prime \prime}(\omega) \sim \omega^{m}, \\
\left(0<m<1, \omega \ll \omega_{\mathrm{p}}\right),
\end{gathered}
$$

where $\chi^{\prime}(0)$ is the static polarization and $\omega_{\mathrm{p}}$ the loss-peak frequency, is observed over many decades of frequency. Expressions (1) and (2) serve as the definition of the universal response behavior.

Note that a consequence of the power laws is that the ratio of the imaginary to the real component of the susceptibility is independent of frequency. The frequency dependence given by equation (1) implies that the real and imaginary components of the complex susceptibility $\tilde{\chi}(\omega)=\chi^{\prime}(\omega)-\mathrm{i} \chi^{\prime \prime}(\omega)$ obey at high frequencies the relation

$$
\frac{\chi^{\prime \prime}(\omega)}{\chi^{\prime}(\omega)}=\cot \left(\frac{\pi n}{2}\right), \quad\left(\omega \gg \omega_{\mathrm{p}}\right)
$$

The experimental behavior of equation (2) leads to a similar frequency-independent rule for the low frequency polarization decrement:

$$
\frac{\chi^{\prime \prime}(\omega)}{\chi^{\prime}(0)-\chi^{\prime}(\omega)}=\tan \left(\frac{\pi m}{2}\right), \quad\left(\omega \ll \omega_{\mathrm{p}}\right) .
$$

This being a unique consequence of Kramers-Kronig relations and does not depend on any particular physical process.

\section{Fractional equations for laws of universal response}

For the region $\omega \gg \omega_{\mathrm{p}}$, the universal fractional power law (1) can be presented in the form

$$
\tilde{\chi}(\omega)=\chi_{\alpha}(\mathrm{i} \omega)^{-\alpha}, \quad(0<\alpha<1)
$$


with some positive constant $\chi_{\alpha}$ and $\alpha=1-n$. Using

$$
(\mathrm{i} \omega)^{\alpha}=|\omega|^{\alpha} \exp \{\mathrm{i} \alpha \pi \operatorname{sgn}(\omega) / 2\}
$$

it is easy to derive relation (3). The polarization density can be written as

$$
\begin{aligned}
& \mathbf{P}(t, r)=\mathcal{F}^{-1}(\tilde{\mathbf{P}}(\omega, r))=\varepsilon_{0} \mathcal{F}^{-1}(\tilde{\chi}(\omega) \tilde{\mathbf{E}}(\omega, r)) \\
& \quad=\varepsilon_{0} \chi_{\alpha} \mathcal{F}^{-1}\left((\mathrm{i} \omega)^{-\alpha} \tilde{\mathbf{E}}(\omega, r)\right),
\end{aligned}
$$

where $\tilde{\mathbf{P}}(\omega, r)$ is a Fourier transform $\mathcal{F}$ of $\mathbf{P}(t, r)$. Equation (6) can be represented by integrals of noninteger order $\alpha=1-n$. The fractional Liouville integral $[10,11]$ is defined by

$$
\left(I_{+}^{\alpha} f\right)(t)=\frac{1}{\Gamma(\alpha)} \int_{-\infty}^{t} \frac{f\left(t^{\prime}\right) \mathrm{d} t^{\prime}}{\left(t-t^{\prime}\right)^{1-\alpha}} .
$$

The Fourier transform $\mathcal{F}$ of this integral is given (see theorem 7.1. in [10] and theorem 2.15 in [11]) by the relation

$$
\left(\mathcal{F} I_{+}^{\alpha} f\right)(\omega)=\frac{1}{(\mathrm{i} \omega)^{\alpha}}(\mathcal{F} f)(\omega)
$$

As a result, the fractional power law (5) gives

$$
\mathbf{P}(t, r)=\varepsilon_{0} \chi_{\alpha}\left(I_{+}^{\alpha} \mathbf{E}\right)(t, r), \quad(0<\alpha<1) .
$$

For the region $\omega \ll \omega_{\mathrm{p}}$, the universal fractional power law (2) can be presented as

$$
\tilde{\chi}(\omega)=\tilde{\chi}(0)-\chi_{\beta}(\mathrm{i} \omega)^{\beta}, \quad(0<\beta<1)
$$

with some positive constants $\chi_{\beta}, \tilde{\chi}(0)$, and $\beta=m$. It is not hard to prove that equation (4) is satisfied. The law (8) can be presented by the fractional Liouville derivative $[10,11]$ that is denoted by $D_{+}^{\beta}$. The differential operator $D_{+}^{\beta}$ of order $\beta$ is defined by the equation

$$
\begin{aligned}
& \left(D_{+}^{\beta} f\right)(t)=\frac{\partial^{k}}{\partial t^{k}}\left(I_{+}^{k-\beta} f\right)(t)=\frac{1}{\Gamma(k-\beta)} \\
& \times \frac{\partial^{k}}{\partial t^{k}} \int_{-\infty}^{t} \frac{f\left(t^{\prime}\right) \mathrm{d} t^{\prime}}{\left(t-t^{\prime}\right)^{\beta-k+1}}, \quad(k-1<\beta<k) .
\end{aligned}
$$

The Fourier transforms $\mathcal{F}$ of this derivative (see theorem 7.1. in [10] and theorem 2.15 in [11]) is given by

$$
\left(\mathcal{F} D_{+}^{\beta} f\right)(\omega)=(\mathrm{i} \omega)^{\beta}(\mathcal{F} f)(\omega)
$$

As a result, the fractional power law (8), gives the polarization density

$$
\mathbf{P}(t, r)=\varepsilon_{0} \mathcal{F}^{-1}(\tilde{\chi}(\omega) \tilde{\mathbf{E}}(\omega, r))
$$

in the form

$$
\begin{aligned}
\mathbf{P}(t, r) & =\varepsilon_{0} \tilde{\chi}(0) \mathbf{E}(t, r)-\varepsilon_{0} \chi_{\beta}\left(D_{+}^{\beta} \mathbf{E}\right)(t, r), \\
(0<\beta<1) . &
\end{aligned}
$$

Equations (7) and (11) can be considered as the universal response laws [4-6] for the time domain. These equations allow us to derive fractional equations for electric and magnetic fields.

\section{Fractional equations of the Curie-von Schweidler law}

Using (7) and (11), the polarization current density

$$
\mathbf{J}_{\mathrm{pol}}(t, r)=\frac{\partial \mathbf{P}(t, r)}{\partial t}
$$

can be described by the fractional equations

$$
\mathbf{J}_{\mathrm{pol}}(t, r)=\varepsilon_{0} \chi_{\alpha}\left(D_{+}^{1-\alpha} \mathbf{E}\right)(t, r), \quad(0<\alpha<1),
$$

and

$$
\begin{aligned}
& \mathbf{J}_{\mathrm{pol}}(t, r)=\varepsilon_{0} \tilde{\chi}(0) D_{t}^{1} \mathbf{E}(t, r)-\varepsilon_{0} \chi_{\beta}\left(D_{+}^{1+\beta} \mathbf{E}\right)(t, r), \\
& \quad(0<\beta<1) .
\end{aligned}
$$

For constant electric field $\mathbf{E}(t, r)$, equations (13) and (14) show that the time dependence of the relaxation of the polarization current density (12) after the sudden removal of a polarizing field follows the power laws, which is widely observed in practice [4] and is known as the Curie-von Schweidler law $[12,13]$. For the changeable field $\mathbf{E}(t, r)$, equations (13) and (14) can be considered as a generalization of the wellknown Curie-von Schweidler law. Let us consider some examples of this generalization.

(1) Using (13) and (14), we can derive the usual Curievon Schweidler law. The most elementary part of the applied field $\mathbf{E}(t, r)$ is the step function

$$
\mathbf{E}(t, r)= \begin{cases}0, & t<a, \\ \mathbf{E}_{0}(r), & t>a .\end{cases}
$$

In this case, equations (13) and (14) give

$$
\begin{aligned}
& \mathbf{J}_{\mathrm{pol}}(t, r)=\varepsilon_{0} \chi_{\alpha} \mathbf{E}_{0}(r)\left({ }_{a} D_{t}^{1-\alpha} 1\right)(t) \\
& =\varepsilon_{0} \chi_{\alpha} \mathbf{E}_{0}(r) \frac{(t-a)^{\alpha-1}}{\Gamma(\alpha)}, \quad(t>a, 0<\alpha<1),
\end{aligned}
$$

and

$$
\begin{aligned}
& \mathbf{J}_{\mathrm{pol}}(t, r)=-\varepsilon_{0} \chi_{\beta} \mathbf{E}_{0}(r)\left({ }_{a} D_{t}^{1+\beta} 1\right)(t) \\
& =-\varepsilon_{0} \chi_{\beta} \mathbf{E}_{0}(r) \frac{(t-a)^{-\beta-1}}{\Gamma(-\beta)}, \quad(t>a, 0<\beta<1),
\end{aligned}
$$

where we use [10] the relation

$$
\left({ }_{a} D_{t}^{\alpha} 1\right)(t)=\frac{(t-a)^{-\alpha}}{\Gamma(1-\alpha)}, \quad(t>a, \alpha>0) .
$$

Here ${ }_{a} D_{t}^{\alpha}$ is the fractional derivative

$$
\begin{aligned}
& \left({ }_{a} D_{t}^{\alpha} u\right)(t)=\frac{\partial^{k}}{\partial t^{k}}\left({ }_{a} I_{t}^{k-\alpha} u\right)(t) \\
& =\frac{1}{\Gamma(k-\alpha)} \frac{\partial^{k}}{\partial t^{k}} \int_{a}^{t} \frac{u\left(t^{\prime}\right) \mathrm{d} t^{\prime}}{\left(t-t^{\prime}\right)^{\alpha-k+1}},
\end{aligned}
$$

where $n=[\operatorname{Re}(\alpha)]+1$, i.e., $n-1<\alpha<n$.

As a result, we obtain the usual Curie-von Schweidler law that is described by

$$
\begin{aligned}
& \mathbf{E}(t, r)=\varepsilon_{0} \chi_{\alpha} \mathbf{E}_{0}(r) \frac{(t-a)^{\alpha-1}}{\Gamma(\alpha)}, \quad(t>a, 0<\alpha<1) ; \\
& \mathbf{E}(t, r)=-\varepsilon_{0} \chi_{\beta} \mathbf{E}_{0}(r) \frac{(t-a)^{-\beta-1}}{\Gamma(-\beta)}, \quad(t>a, 0<\beta<1) .
\end{aligned}
$$


(2) The experimental applied field $\mathbf{E}(t, r)$ can be presented as

$$
\mathbf{E}(t, r)=\mathbf{E}_{0}(r) \sin (\lambda t) .
$$

Using the relation [10]:

$$
D_{+}^{\alpha} \sin (\lambda t+\phi)=\lambda^{\alpha} \sin (\lambda t+\phi+\alpha \pi / 2), \quad(\alpha>0),
$$

equations (13), (14), and (19) give

$$
\begin{aligned}
& \mathbf{J}_{\mathrm{pol}}(t, r)=\varepsilon_{0} \chi_{\alpha} \mathbf{E}_{0}(r) \lambda^{\alpha} \sin (\lambda t+(1-\alpha) \pi / 2), \\
& \quad(0<\alpha<1),
\end{aligned}
$$

and

$$
\begin{aligned}
& \mathbf{J}_{\mathrm{pol}}(t, r)=\varepsilon_{0} \mathbf{E}_{0}(r)[\tilde{\chi}(0) \lambda \cos (\lambda t) \\
& \left.\quad-\chi_{\beta} \lambda^{1+\beta} \sin (\lambda t+(1+\beta) \pi / 2)\right], \quad(0<\beta<1) .
\end{aligned}
$$

Equation (21) can be rewritten in the form

$$
\mathbf{J}_{\mathrm{pol}}(t, r)=\varepsilon_{0} a(\beta) \mathbf{E}_{0}(r) \sin (\lambda t+b(\beta)),
$$

where $a(\beta)$ and $b(\beta)$ describe the amplitude and phase changes by the equations

$$
a(\beta)=\sqrt{A^{2}(\beta)+B^{2}(\beta)}, \quad b(\beta)=\arctan \left(\frac{A(\beta)}{B(\beta)}\right) .
$$

Here

$$
\begin{gathered}
A(\beta)=\tilde{\chi}(0) \lambda-\chi_{\beta} \lambda^{1+\beta} \cos (\beta \pi / 2), \\
B(\beta)=\chi_{\beta} \lambda^{1+\beta} \sin (\beta \pi / 2) .
\end{gathered}
$$

(3) For the applied field

$$
\mathbf{E}(t, r)= \begin{cases}0, & t<a, \\ \mathbf{E}_{0}(r) g(t), & t>a\end{cases}
$$

with some function $g(t)$, exact expressions for the polarization current density $\mathbf{J}_{\text {pol }}(t, r)$ can be derived by using the list of fractional derivatives of the function $g(t)$ (see tables 9.1-9.3 in [10]). For $g(t)=(t-a)^{s}$, where $s>-1$,

${ }_{a} D_{t}^{\alpha} g(t)={ }_{a} D_{t}^{\alpha}(t-a)^{s}=\frac{\Gamma(s+1)}{\Gamma(s+1-\alpha)}(t-a)^{s-\alpha}$,

$$
(s>-1, \alpha>0) \text {. }
$$

The fractional derivative of $g(t)=\cos [\lambda(t-a)]$ is

$$
\begin{aligned}
& { }_{a} D_{t}^{\alpha} \cos \lambda(t-a)=\frac{(t-a)^{-\alpha}}{2 \Gamma(1-\alpha)} \\
& \quad \times\left[{ }_{1} F_{1}(1,1-\alpha, \mathrm{i} \lambda(t-a))+{ }_{1} F_{1}(1,1-\alpha,-\mathrm{i} \lambda(t-a))\right],
\end{aligned}
$$

where ${ }_{1} F_{1}(a, b, c)$ is a hypergeometric function [14]. For $g(t)=\exp (-\lambda t)$, we use

${ }_{a} D^{\alpha} \mathrm{e}^{-\lambda t}=\mathrm{e}^{-\lambda t}(t-a)^{-\alpha} E_{1,1-\alpha}[-\lambda(t-a)]$,

$(0<\alpha<1)$,

where $E_{\alpha, \beta}[z]$ is the Mittag-Leffler function [15]:

$$
E_{\alpha, \beta}[z]=\sum_{k=0}^{\infty} \frac{z^{k}}{\Gamma(\alpha k+\beta)} .
$$

If $\alpha=\beta=1$, then $E_{1,1}[z]=\exp (z)$, where $\Gamma(k+1)=k$ ! for positive integer $k$.

As a result, fractional relations (13) and (14) can be considered as a generalization of the formulation of the Curie-von Schweidler law from a constant electric field into changeable fields $\mathbf{E}(t, r)$.

\section{Fractional Gauss's laws for electric field}

Time-domain laws are presented by the fractional integral and differential equations (7) and (11). Using the equation

$$
\mathbf{D}(t, r)=\varepsilon_{0} \mathbf{E}(t, r)+\mathbf{P}(t, r),
$$

and Gauss's law

$$
\operatorname{div} \mathbf{D}(t, r)=\rho(t, r)
$$

for the electric displacement field $\mathbf{D}(t, r)$, we get

$$
\varepsilon_{0} \operatorname{div} \mathbf{E}(t, r)+\operatorname{div} \mathbf{P}(t, r)=\rho(t, r) .
$$

Substitution of (7) and (11) into (28) gives

$$
\begin{aligned}
& \varepsilon_{0} Z(t, r)+\varepsilon_{0} \chi_{\alpha}\left(I_{+}^{\alpha} Z\right)(t, r)=\rho(t, r), \\
& \quad(0<\alpha<1), \\
& \varepsilon_{0} \tilde{\chi}(0) Z(t, r)-\varepsilon_{0} \chi_{\beta}\left(D_{+}^{\beta} Z\right)(t, r)=\rho(t, r), \\
& \quad(0<\beta<1),
\end{aligned}
$$

where $Z(t, r)=\operatorname{div} \mathbf{E}(t, r)$. Using $\left(D_{+}^{\alpha} I_{+}^{\alpha} Z\right)(t, r)=Z(t, r)$ (see lemma 2.20 in [11]), we obtain

$$
\begin{aligned}
& \left(D_{+}^{\alpha} Z\right)(t, r)+\chi_{\alpha} Z(t, r)=\frac{1}{\varepsilon_{0}}\left(D_{+}^{\alpha} \rho\right)(t, r), \\
& (0<\alpha<1), \\
& \left(D_{+}^{\beta} Z\right)(t, r)-\frac{\tilde{\chi}(0)}{\chi_{\beta}} Z(t, r)=-\frac{1}{\varepsilon_{0} \chi_{\beta}} \rho(t, r), \\
& (0<\beta<1),
\end{aligned}
$$

which are fractional differential equations of Gauss's law for the electric field $\mathbf{E}(t, r)$.

For a fixed (stationary) region $R$ of medium, we define the total electric charge

$$
Q(t)=\int_{R} \rho(t, r) \mathrm{d} V .
$$

The electric field $\mathbf{E}=\mathbf{E}(t, r)$ passing through a surface $S=$ $\partial R$ gives the electric flux

$$
\Phi_{E}(t)=\int_{S}(\mathbf{E}, \mathrm{d} \mathbf{S})=\int_{R} \operatorname{div} \mathbf{E} \mathrm{d} V .
$$

The integration of equations (31) and (32) over the region $R$ gives the fractional equations

$$
\begin{aligned}
& \left(D_{+}^{\alpha} \Phi_{E}\right)(t)+\chi_{\alpha} \Phi_{E}(t)=\frac{1}{\varepsilon_{0}}\left(D_{+}^{\alpha} Q\right)(t), \\
& (0<\alpha<1), \\
& \left(D_{+}^{\beta} \Phi_{E}\right)(t)-\frac{\tilde{\chi}(0)}{\chi_{\beta}} \Phi_{E}(t)=-\frac{1}{\varepsilon_{0} \chi_{\beta}} Q(t), \\
& (0<\beta<1) .
\end{aligned}
$$

These equations represent the integral Gauss's laws for the electric field in dielectric media. Note that $D_{+}^{\alpha}$ is the differential operator of order $\alpha$ that is defined by equation (9). If $\mathbf{E}(t, r)$ is defined by

$$
\mathbf{E}(t, r)= \begin{cases}0, & t<a, \\ \mathbf{E}(t, r), & t>a,\end{cases}
$$


then the operator $D_{+}^{\alpha}$ transforms into the operator ${ }_{a} D_{t}^{\alpha}$ that is defined by equation (17).

Consider equations (35) and (36) in the form

$$
{ }_{a} D_{t}^{\alpha} u(t)-\lambda u(t)=f(t), \quad 0<\alpha<1,
$$

where $u(t)$ presents $Z(t, r)$ or $\Phi_{E}(t) \lambda$ is $-\chi_{\alpha}$ or $\tilde{\chi}(0) / \chi_{\beta}$, the function $f(t)$ is $\left(1 / \varepsilon_{0}\right)_{a} D_{t}^{\alpha} \rho(t, r)$ and $\left(-1 / \varepsilon_{0} \chi_{\beta}\right) \rho(t, r)$, or $\left(1 / \varepsilon_{0}\right)_{a} D_{t}^{\alpha} Q(t)$ and $\left(-1 / \varepsilon_{0} \chi_{\beta}\right) Q(t)$. The fractional derivative ${ }_{a} D_{t}^{\alpha}$ is defined by (17). Note that

$$
\left(D_{+}^{\alpha} u\right)(t)=\lim _{a \rightarrow-\infty}\left({ }_{a} D_{t}^{\alpha} u\right)(t) .
$$

Barrett [16] in 1954 first considered the Cauchy type problem for a linear differential equation (38) with initial conditions

$$
\left({ }_{a} D_{t}^{\alpha-1} u\right)(a)=C,
$$

where ${ }_{a} D_{t}^{\alpha-1}={ }_{a} I_{t}^{1-\alpha}$ is the Riemann-Liouville fractional integral [11]. Note that any change in the past of the input function of a fractional order system changes the future of the solution [17-19]. Hence the past of such systems cannot be represented by a finite set of local conditions [17-19], and the initial conditions have the integral form (39).

Barrett proved in [16] (see also [11] theorem 4.1 and example 4.1.) that if $f(t)$ is an integrable function on $(a, b)$, then the problem (38), (39) has the unique solution given by

$$
\begin{aligned}
u(t) & =C(t-a)^{\alpha-1} E_{\alpha, \alpha}\left[\lambda(t-a)^{\alpha}\right] \\
& +\int_{a}^{t}\left(t-t^{\prime}\right)^{\alpha-1} E_{\alpha, \alpha}\left[\lambda\left(t-t^{\prime}\right)^{\alpha}\right] f\left(t^{\prime}\right) \mathrm{d} t^{\prime},
\end{aligned}
$$

where $E_{\alpha, \alpha}[z]$ is the Mittag-Leffler function defined by (26). For $f(t)=0$, we obtain

$$
u(t)=C(t-a)^{\alpha-1} E_{\alpha, \alpha}\left[\lambda(t-a)^{\alpha}\right] .
$$

To consider the asymptotic behavior of the solutions (40) and (41), we can use the integral representation [20-22] of the Mittag-Leffler function:

$$
\begin{gathered}
E_{\alpha, \beta}[z]=\frac{1}{2 \pi \mathrm{i} \alpha} \int_{\gamma(a, \delta)} \frac{\mathrm{e}^{\xi^{1 / \alpha}} \xi^{(1-\beta) / \alpha}}{\xi-z} \mathrm{~d} \xi, \\
(1<\alpha<2),
\end{gathered}
$$

where $\pi \alpha / 2<\delta<\min \{\pi, \pi \alpha\}$. The contour $\gamma(a, \delta)$ consists of two rays $S_{-\delta}=\{\arg (\xi)=-\delta,|\xi| \geqslant a\}$ and $S_{+\delta}=\{\arg (\xi)=+\delta,|\xi| \geqslant a\}$, and a circular $\operatorname{arc} C_{\delta}=\{|\xi|=$ $1,-\delta \leqslant \operatorname{arc}(\xi) \leqslant \delta\}$. Let us denote the region on the left from $\gamma(a, \delta)$ by $G^{-}(a, \delta)$. Then the asymptote of (42) has the form $[21,22]$ :

$$
\begin{aligned}
E_{\alpha, \beta}[z] & =-\sum_{k=1}^{\infty} \frac{z^{-k}}{\Gamma(\beta-\alpha k)}, \quad z \in G^{-}(a, \delta), \\
(|z| & \rightarrow \infty)
\end{aligned}
$$

and $\delta \leqslant|\arg (z)| \leqslant \pi$. In our case, $z=\lambda(t-a)^{\alpha}, \arg (z)=\pi$. As a result, we arrive at the asymptote of the solution, which exhibits power-like tails. These power-like tails are the most important effect of the noninteger derivative in the fractional equations.

\section{Conclusion}

In this paper, it has been shown that the electromagnetic fields in a wide class of dielectric materials must be described by differential equations of fractional order with respect to time. The orders of these equations are defined by exponents of the 'universal' response laws for frequency dependence of the dielectric susceptibility. A remarkable property of the dynamics described by the fractional equations for electromagnetic fields is that the solutions have power-like tails. The typical features of the 'universal' electromagnetic phenomenon and the suggested fractional equations are common to a wide class of materials, regardless of the type of physical structure, chemical composition or of the nature of the polarizing species, whether dipoles, electrons or ions.

For small fractionality $\alpha$ (or $\beta$ ), it is possible to use a $\varepsilon$-expansion [23] over the small parameter. The suggested fractional differential equations, which describe the fields in dielectric media with power-law frequency-domain response, can be solved numerically. There are several numerical methods to solve fractional equations (for example, see [10, 24-26]). In this paper we consider only general equations without numerical examples.

The presented approach does not take into account the dielectric dispersion region around the loss-peak frequency $\omega_{\mathrm{p}}$. This is a limitation of the fractional calculus formalism. Note that the universal dispersion is only valid over a finite frequency region. There exist numerical methods without a priori assumptions to tackle frequency to time and time to frequency conversions using the distribution of relaxation times approach. There are several papers on this topic by different authors (for example, see [27-31]).

Note that Tuncer has shown [32] that in binary mixtures Maxwell-Wagner polarization could lead to a universal dispersion on a finite frequency region. In that study the disorder in the system leads to a universal dispersion through the Gauss law.

Note that it will be interesting to find a generalized physical explanation for fractional power laws. Unfortunately, after more than 30 years we still do not have an explanation of the universal response from first principles. There are several papers on the fractional kinetic approach to the dielectric universal response by different authors (for example, see [33-35]).

\section{References}

[1] Debye P 1912 Some results of kinetic theory of isolators. Preliminary announcement Phys. Z. 13 97-100

[2] Debye P and Huckel E 1923 The theory of electrolytes I. The lowering of the freezing point and related occurrences Phys. Z. 24 185-206

[3] Debye P 1945 Polar Molecules (New York: Dover) 
[4] Jonscher A K 1983 Dielectric Relaxation in Solids (London: Chelsea Dielectrics Press)

[5] Jonscher A K 1996 Universal Relaxation Law (London: Chelsea Dielectrics Press)

[6] Jonscher A K 1999 Dielectric relaxation in solids J. Phys. D: Appl. Phys. 32 R57-70

[7] Ramakrishnan T V and Lakshmi M R (ed) 1984 Non-Debye Relaxation in Condensed Matter (Singapore: World Scientific)

[8] Jonscher A K 1977 Universal dielectric response Nature $267673-9$

Jonscher A K 1978 Low-frequency dispersion in carrier-dominated dielectrics Phil. Mag. B 38 587-601

[9] Ngai K L, Jonscher A K and White C T 1979 Origin of the universal dielectric response in condensed matter Nature 277 185-9

[10] Samko S G, Kilbas A A and Marichev O I 1993 Fractional Integrals and Derivatives Theory and Applications (New York: Gordon and Breach)

[11] Kilbas A A, Srivastava H M and Trujillo J J 2006 Theory and Application of Fractional Differential Equations (Amsterdam: Elsevier)

[12] Curie J 1889 Recherches sur le pouvoir inducteur specifique et la conductibilite des corps cristallises Ann. Chim. Phys. 17 385-434

Curie J 1989 Recherches sur la conductibilite des corps cristallises Ann. Chim. Phys. 18 203-69 (in French)

[13] von Schweidler E 1907 Studien ber anomalien im verhalten der dielektrika (Studies on the anomalous behaviour of dielectrics) Ann. Phys., Lpz. 24 711-70 Series 4 (in German)

[14] Erdelyi A, Magnus W, Oberhettinger F and Tricomi F G 1981 Higher Transcendental Functions vol 1 (Melbbourne, FL: Krieger)

[15] Gorenflo R, Kilbas A A and Rogosin S V 1998 On the generalized Mittag-Leffler type functions Integral Transform. Spec. Funct. 7 215-24

[16] Barrett J H 1954 Differential equations of non-integer order Can. J. Math. 6 529-41

[17] Kempfle S and Schafer I 2000 Fractional differential equations and initial conditions Fractional Calc. Appl. Anal. 3 $387-400$

[18] Fukunaga M and Shimizu N 2004 Role of prehistories in the initial value problems of fractional viscoelastic equations Nonlinear Dyn. 38 207-20

[19] Heymans N and Podlubny I 2006 Physical interpretation of initial conditions for fractional differential equations with Riemann-Liouville fractional derivatives Rheol. Acta $45765-71$
[20] Podlubny I 1999 Fractional Differential Equations (San Diego, CA: Academic)

[21] Gorenflo R, Loutchko J and Luchko Y 2002 Computation of the Mittag-Leffler function and its derivative Fractional Calc. Appl. Anal. 5 491-518

[22] Mainardi F and Gorenflo R 2000 On Mittag-Leffler-type functions in fractional evolution processes J. Comput. Appl. Math. 118 283-99

[23] Tarasov V E and Zaslavsky G M 2006 Dynamics with low-level fractionality Physica A $\mathbf{3 6 8} 399-415$

[24] Gorenflo R 1997 Fractional calculus: some numerical methods Fractals and Fractional Calculus in Continuum Mechanics ed A Carpinteri and F Mainardi (Wien: Springer) pp 277-90

[25] Agrawal O P 2002 Solution for a fractional diffusion-wave equation defined in a bounded domain Nonlinear Dyn. 29 145-55

[26] Tadjeran C, Meerschaert M M and Scheffler H P 2006 A second-order accurate numerical approximation for the fractional diffusion equation J. Comput. Phys. 213 205-13

[27] Tuncer E and Macdonald J R 2006 Comparison of methods for estimating continuous distributions of relaxation times J. Appl. Phys. 99074106

[28] Macdonald J R and Tuncer E 2007 Deconvolution of immittance data: some old and new methods J. Electroanal. Chem. 602 255-62

[29] Kuhn M and Kliem H 2007 Time-variance of interacting dipole systems Phys. Status Solidi b 244 1418-28

[30] Kuhn M and Kliem H 2006 Modelling non-exponential polarization relaxations in interacting dipole systems Phys. Status Solidi b 243 2913-28

[31] Farag N, Holten S, Wagner A and Kliem H 2003 Numerical transformations of wide-range time- and frequency-domain relaxational spectra IEE Proc. Sci. Meas. Technol. 150 65-74

[32] Tuncer E 2004 Signs of low frequency dispersions in disordered binary dielectric mixtures (fifty-fifty) J. Phys. D: Appl. Phys. 37 334-42

[33] Nigmatullin R R and Nelson S O 2006 Recognition of the fractional kinetics in complex systems: dielectric properties of fresh fruits and vegetables from 0.01 to $1.8 \mathrm{GHz}$ Signal Process. 86 2744-59

[34] Nigmatullin R R, Arbuzov A A, Salehli F, Giz A, Bayrak I and Catalgil-Giz H 2007 The first experimental confirmation of the fractional kinetics containing the complex-power-law exponents: dielectric measurements of polymerization reactions Physica B 388 418-34

[35] Novikov V V and Privalko V P 2001 Temporal fractal model for the anomalous dielectric relaxation of inhomogeneous media with chaotic structure Phys. Rev. E 64031504 